Meta

Journal des traducteurs

Translators' Journal

\title{
Traductologie et sciences cognitives : une dialectique prometteuse
}

\section{Périclès Papavassiliou}

Volume 52, numéro 1, mars 2007

Traductologie : une science cognitive

URI : https://id.erudit.org/iderudit/014717ar

DOI : https://doi.org/10.7202/014717ar

Aller au sommaire du numéro

\section{Éditeur(s)}

Les Presses de l'Université de Montréal

\section{ISSN}

0026-0452 (imprimé)

1492-1421 (numérique)

Découvrir la revue

Citer cet article

Papavassiliou, P. (2007). Traductologie et sciences cognitives : une dialectique prometteuse. Meta, 52(1), 29-36. https://doi.org/10.7202/014717ar

\section{Résumé de l'article}

La traductologie est actuellement de plus en plus conçue comme un domaine interdisciplinaire de recherche fondamentale et appliquée. La traduction, dans le cadre de la théorie interprétative, est considérée comme un processus complexe de communication.

Les mécanismes neurophysiologiques impliqués dans l'activité traduisante sont peu connus à l'heure actuelle. Une approche d'investigation de ces processus est de tenter d'obtenir des résultats expérimentaux convergents par des techniques psycholinguistiques et d'imagerie fonctionnelle de l'activité cérébrale. Dans cet ordre d'idées, des travaux réalisés récemment (dans le cadre de la collaboration entre l'Université de Caen-Basse Normandie - MRSH et l'Université Ionienne - DLETI) sur le fonctionnement de l'attention et de la mémoire de lecture au cours de l'activité traduisante par oculométrie tendent à attribuer un rôle important du buffer épisodique dans le processus de traduction. D'autre part, des futurs travaux de recherche sur les mécanismes neuropsychologiques mis en jeu par ce processus en utilisant des techniques d'imagerie fonctionnelle cérébrale (TEP, IRMf), sur la base de travaux déjà réalisés pour l'étude du bilinguisme et de la traduction de mots ou de phrases élémentaires, pourraient contribuer à éclaircir nos connaissances des mécanismes cognitifs intervenant dans cette activité mentale hautement complexe.
Ce document est protégé par la loi sur le droit d'auteur. L'utilisation des services d’Érudit (y compris la reproduction) est assujettie à sa politique d'utilisation que vous pouvez consulter en ligne.

https://apropos.erudit.org/fr/usagers/politique-dutilisation/ 


\title{
Traductologie et sciences cognitives: une dialectique prometteuse
}

\author{
PÉRICLÈS PAPAVASSILIOU \\ Université ionienne, Corfou, Grèce \\ papabas@otenet.gr
}

\begin{abstract}
RÉSUMÉ
La traductologie est actuellement de plus en plus conçue comme un domaine interdisciplinaire de recherche fondamentale et appliquée. La traduction, dans le cadre de la théorie interprétative, est considérée comme un processus complexe de communication.

Les mécanismes neurophysiologiques impliqués dans l'activité traduisante sont peu connus à l'heure actuelle. Une approche d'investigation de ces processus est de tenter d'obtenir des résultats expérimentaux convergents par des techniques psycholinguistiques et d'imagerie fonctionnelle de l'activité cérébrale. Dans cet ordre d'idées, des travaux réalisés récemment (dans le cadre de la collaboration entre l'Université de Caen-Basse Normandie - MRSH et l'Université lonienne - DLETI) sur le fonctionnement de l'attention et de la mémoire de lecture au cours de l'activité traduisante par oculométrie tendent à attribuer un rôle important du buffer épisodique dans le processus de traduction. D'autre part, des futurs travaux de recherche sur les mécanismes neuropsychologiques mis en jeu par ce processus en utilisant des techniques d'imagerie fonctionnelle cérébrale (TEP, IRMf), sur la base de travaux déjà réalisés pour l'étude du bilinguisme et de la traduction de mots ou de phrases élémentaires, pourraient contribuer à éclaircir nos connaissances des mécanismes cognitifs intervenant dans cette activité mentale hautement complexe.
\end{abstract}

\begin{abstract}
Translatology, conceived as a complex communicative process, has to be addressed as an interdisciplinary field of scientific research. Such research activities should comprise, apart from stylistics and contrastive linguistics, a cognitive science-based approach. Research tools and methodology developed by cognitive psychology, psycholinguistics and neurophysiology - especially during the last decade - gave promising results for further development.
\end{abstract}

\section{$\Pi \varepsilon \rho i \lambda \eta \Psi \eta$}

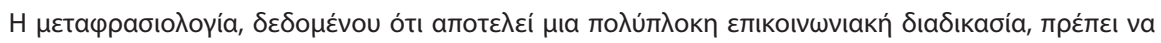

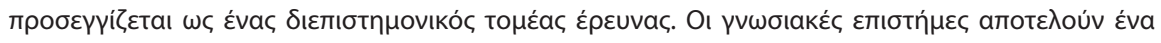

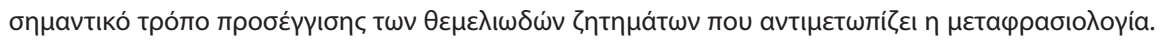

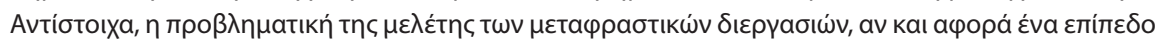

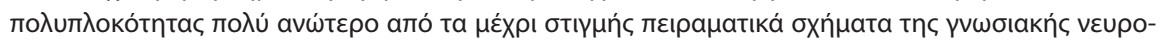

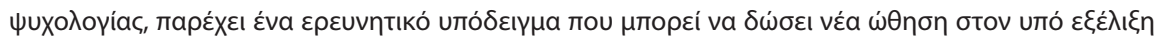

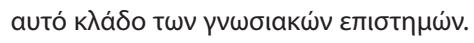

\section{MOTS-CLÉS/KEYWORDS}

traductologie, sciences cognitives, imagerie fonctionnelle cérébrale, bilinguisme

Dans le cadre de la théorie interprétative de la traduction, le traducteur fonctionne dans le sens de la facilitation de la communication entre l'auteur du texte de départ 
et des lecteurs potentiels qui n'ont pas la possibilité d'avoir accès au contenu du texte à cause du manque de connaissance total ou partiel de la langue source. Dans ce sens, le but fondamental de l'acte de traduction est de créer, sur la base du message contenu dans le texte de départ, un texte en langue d'arrivée transmettant un message équivalent. Le traducteur fait donc fonction d'une interface entre les deux formes du message. Par conséquent, pour remplir ce rôle, le traducteur doit au préalable comprendre et assimiler le message de l'auteur. C'est dans ce sens que l'on peut énoncer de manière liminaire que traduire, c'est comprendre dans le but de permettre à d'autres de comprendre.

Sous cet angle, toute connaissance et capacité linguistique du traducteur (comme du reste de tout lecteur exigeant d'un texte) est une condition nécessaire mais non suffisante pour la compréhension du message contenu dans le texte. Il ne suffit pas de reconnaître les mots pour saisir le sens d'un énoncé. Pour arriver à un tel résultat, il faut réaliser la concordance fructueuse du bagage cognitif du lecteur-traducteur avec ses capacités linguistiques.

Mais à partir de quel moment, sur la base de quels critères peut-on considérer qu'on est effectivement arrivé à la compréhension du contenu d'un texte? En fait, il est difficile d'obtenir de tels critères objectifs. On pourrait néanmoins considérer comme critère d'une compréhension suffisante la formation d'une image mentale claire (ou bien, pour reprendre la terminologie de la psychologie cognitive, d'une représentation mentale) du message véhiculé par le texte. C'est essentiellement sur ce point crucial que la traductologie rejoint une problématique fondamentale soulevée par les sciences cognitives. Je pense qu'il serait pertinent d'évoquer ici la discussion théorique au sein de la communauté des scientifiques travaillant dans le domaine des sciences cognitives à propos de la nature même de ce concept de représentation: doiton lui attribuer un contenu purement sémantique, ou bien possède-t-il une dimension épistémologique ou ontologique qui correspond à une vision théorique globale des mécanismes perceptuels, linguistiques et cognitifs? Je n'entrerai pas ici dans une telle discussion, d'une part, car je ne suis pas spécialiste de ces domaines, et d'autre part, car on dépasserait ainsi largement l'objet de cette intervention. Il me semble toutefois utile de tenir compte dans notre démarche de l'intérêt de cette réflexion sur l'essence même du concept de représentation, car il est directement impliqué dans la vision théorique de l'activité traduisante.

Depuis les années 1980 s'est amorcée une réorientation de la recherche en traductologie, processus qui est en plein développement. Un axe essentiel de ce développement est lié à une conception processive, dynamique de la fonctionnalité du texte qui met l'accent sur l'interaction entre le cerveau et le texte. Dans le cadre de cette approche, le sens n'est pas conçu comme une entité statique qui est à extraire du texte, mais plutôt comme une entité dynamique qui prend forme au cours de l'effort du traducteur de détecter et d'appréhender au niveau cognitif son contenu, pour ensuite le reformuler - surtout dans ses composantes sémantiques et pragmatologiques - dans le texte d'arrivée. Ainsi, les étapes du processus de traduction (compréhension du texte de départ, médiation culturelle et linguistique, mise en forme du texte d'arrivée) ne sont plus considérées comme une simple séquence de décodage et de recodage de messages, mais comme des processus complexes d'inférence et de restructuration. Par voie de conséquence, la recherche dans le domaine de la traductologie a été en grande partie orientée vers les sciences cognitives (ce qui fut dans un premier temps évoqué 
par la fameuse formulation: que se passe-t-il dans la boîte noire du traducteur?). Comme résultat de cette réorientation, des éléments de modèles provenant de la psychologie cognitive, de la psycholinguistique et de la neurophysiologie ont été intégrés dans les travaux de recherche en traductologie. Ainsi donc, la recherche traductologique fut en grande partie recentrée de la linguistique contrastive et la stylistique vers les sciences cognitives.

Il est effectivement pertinent de se demander comment le traducteur réalise la liaison entre l'architecture du texte et le système socioculturel, et en quoi diffère à ce niveau l'approche d'un traducteur débutant par rapport à celle d'un traducteur expérimenté. La réponse à ce type de problématique ne peut pas se résumer à un simple processus de résolution de problèmes au niveau linguistique, métalinguistique, textuel et métatextuel; il s'agit en effet d'un niveau supérieur de prise de décision, qui présuppose une capacité de localisation des difficultés et de leur résolution de manière appropriée, afin d'arriver à proposer des solutions efficaces. Ce processus global est directement lié aux mécanismes régissant le fonctionnement du cerveau et de l'organisme dans son ensemble, qui sont le support de la perception en tant que processus cognitif.

Notons d'emblée que, au stade actuel du développement des sciences cognitives et en particulier de la psychologie cognitive, les mécanismes qui conduisent à la formation des représentations ne sont pas encore suffisamment connus. Les sciences cognitives constituent un domaine interdisciplinaire ayant comme objet l'étude des processus cognitifs et globalement de l'intelligence. Elles reposent sur des domaines scientifiques comme la psychologie, la neuropsychologie, les neurosciences, la linguistique, l'anthropologie, l'intelligence artificielle, l'informatique, la philosophie. L'approche actuelle des sciences cognitives est fondée sur l'idée que l'intelligence humaine peut être conçue en termes de structures représentatives, qui servent de support à des processus de type computationnel. Néanmoins, le débat est toujours ouvert pour savoir dans quelle mesure les processus intervenant au niveau du cerveau, et qui sont directement liés à la nature même de l'intelligence humaine, sont en réalité du même type que ceux qui ont lieu dans les ordinateurs. Comme pour savoir ce que signifie exactement ce que l'on appelle représentation mentale dans le cerveau humain et quel est son rapport avec la structure et le fonctionnement de l'ordinateur. Ces points ne sont pas encore suffisamment clarifiés, dans la mesure où notre compréhension du mode de fonctionnement du cerveau ne permet pas encore d'y parvenir.

À partir des années 1960, nous assistons au développement d'une étude systématique de la corrélation entre les structures du cerveau et le comportement, le but étant d'analyser les processus d'activation, de contrôle et d'inhibition au niveau de cerveau au moment même où ils interviennent, que ce soit dans des cas pathologiques ou chez des sujets sains. Sur cette base est apparue la neuropsychologie cognitive, qui doit en grande partie son développement à l'évolution des sciences biologiques et des technologies d'investigation expérimentale. L'ensemble des données de la psychophysiologie - notamment en ce qui concerne le domaine cognitif - soulignent l'importance du substrat neuronal pour l'élaboration des conduites. Les activités couramment qualifiées de psychiques mettent en jeu des structures nerveuses différenciées, en fonction de leur degré de complexité. Elles impliquent un schéma dont le fonctionnement global est identique, et qui consiste en l'activation d'entités spécialisées soit en périphérie de l'organisme, soit au niveau central, soit encore au niveau des connexions qui 
délimitent des réseaux organisés permettant l'élaboration de réponses spécifiques. Rappelons ici que le système nerveux humain n'est pas limité au cerveau, mais constitue un réseau spécialisé et diversifié couvrant l'ensemble de l'organisme et permettant la propagation de l'influx nerveux, et par ce biais la transmission et la gestion des informations.

Toute étude ayant comme objet des caractéristiques fonctionnelles du cerveau ne peut se limiter à une méthodologie statique, car l'objet n'est pas de localiser d'hypothétiques centres spécialisés, mais plutôt des modalités de fonctionnement. On parle dans ce cas d'une approche de localisation fonctionnelle, rendue possible essentiellement grâce au développement à partir des années 1970 des techniques d'imagerie fonctionnelle. Il s'agit de techniques expérimentales permettant l'observation du cerveau en plein fonctionnement, grâce à des systèmes d'enregistrement d'indications fonctionnelles, de construction par ordinateur d'images reflétant les intensités constatées et de leur transcription sous forme de cartes fonctionnelles.

Les techniques d'imagerie fonctionnelle sont basées essentiellement sur les propriétés biochimiques et physiologiques des cellules nerveuses (métabolisme, activité électrique). Il s'agit notamment de la tomographie par émission de positons (TEP) et de l'imagerie par résonance magnétique fonctionnelle (IRMf), qui vient s'ajouter aux techniques électro-encéphalographiques plus classiques. Ces deux techniques d'imagerie du cerveau ne doivent pas être considérées comme équivalentes; chacune possède des caractéristiques particulières, qui sont à prendre en considération afin d'aboutir à une interprétation fructueuse des données obtenues. En effet, ces techniques se différencient au niveau de leur pouvoir de résolution, qui correspond en première approximation à la précision de la description de l'activité fonctionnelle étudiée. La performance de chacune de ces techniques est estimée par rapport à deux critères de référence: la résolution spatiale et la résolution temporelle. Étant donné leur niveau élevé de complexité, les activités cognitives mobilisent un grand nombre de composantes du système nerveux, sur des durées qui peuvent varier de quelques millisecondes à plusieurs années. Une technique idéale d'imagerie serait donc celle qui permettrait l'étude simultanée de l'activité de très petites zones cérébrales bien délimitées (ce qui correspond à un haut pouvoir de résolution spatiale) pour une durée potentiellement très courte (ce qui correspond à un pouvoir de résolution temporelle élevé). Malheureusement, à l'heure actuelle nous ne disposons pas encore d'une telle technique. Les chercheurs sont donc amenés à choisir la technique utilisée en fonction de la nature du problème à résoudre, du but recherché. Les groupes de recherche qui étudient sur le plan expérimental les processus cognitifs utilisent donc des approches et des techniques différentes, ce qui aboutit en définitive à une complémentarité fructueuse de leurs efforts. On peut d'ores et déjà faire le pari que pendant les années à venir nous assisterons à des progrès significatifs dans le domaine de la technologie de l'imagerie fonctionnelle, ce qui permettra d'obtenir des images de l'activité cérébrale bien plus précises. Cette approche illustre bien le caractère complémentaire de la recherche scientifique dans des domaines comme la neurobiologie, la neuropsychologie et la psychologie cognitive, qui permet d'avancer dans l'étude de processus cognitifs comme l'apprentissage ou la compréhension. Et c'est bien dans ce cadre que l'on peut facilement concevoir tout l'apport que les sciences cognitives peuvent offrir à la traductologie, notamment par l'approche expérimentale du processus de la traduction. 
Notons d'emblée que, en ce qui concerne l'étude du fonctionnement du cerveau au cours de l'activité cérébrale hautement complexe que constitue l'activité traduisante, les études expérimentales réalisées jusqu'à présent en neuropsychologie cognitive sont relativement restreintes. Une première direction intéressante à ce sujet concerne l'étude du fonctionnement de l'attention et de la mémoire du traducteur (mémoire à court terme et mémoire à long terme) dont le rôle décisif au cours de l'opération traduisante est généralement admis. À cet égard, le modèle de structure et de fonctionnement de la mémoire de travail proposé récemment par Baddeley peut être considéré comme un point de référence. Selon ce modèle, la mémoire de travail est constituée de quatre composantes: l'administrateur central (contrôle attentionnel de surveillance, contrôle et coordination de l'ensemble), la boucle phonologique (traitement de l'information phonologique et linguistique), le calepin visuo-spatial (traitement des stimuli visuels et spatiaux, construction et traitement des représentations mentales) et le buffer épisodique (combinaison des différents stimuli, interface entre les informations stockées en mémoire à court terme et en mémoire à long terme). Dans cette direction, une étude expérimentale originale a été effectuée récemment par Aleka Kosma ( Le fonctionnement spécifique de la mémoire de travail dans la traduction», mémoire du master conjoint «Sciences de la traduction - Traductologie et sciences cognitives», Université de Caen-Basse Normandie et Université ionienne, 2004/2005). La technique utilisée dans ces travaux fut l'enregistrement et l'analyse des mouvements oculaires du traducteur grâce à un système électronique et computationnel approprié (ASL Eye Tracking Computer System). Ce travail ayant fait l'objet de la communication précédente, je me limite simplement à souligner le fait que les résultats préliminaires semblent attribuer au buffer épisodique un rôle important dans le processus de traduction. On peut s'attendre à ce que le développement futur des travaux de recherche dans cette direction donnera des résultats intéressants pour la compréhension du mode d'intervention de la mémoire au cours de l'opération traduisante.

Un autre axe de recherche expérimentale en vue d'une meilleure connaissance des mécanismes cérébraux mis en jeu lors de l'activité traduisante est celui mettant à profit des techniques d'imagerie, déjà consacrées en tant qu'outils importants dans l'investigation expérimentale des processus cognitifs au niveau neurobiologique. Les mécanismes cérébraux par lesquels les sujets bilingues peuvent passer d'une langue à l'autre, et traduire d'une langue à l'autre, sont peu connus à l'heure actuelle. Une voie de recherche dans cette direction serait d'essayer d'obtenir des résultats convergents par des approches expérimentales comme les études psycholinguistiques et de neuroimagerie fonctionnelle.

Selon les études de Patterson et Shewell (1987: 273-294), Levelt (1989) et Votaw (1992: 299-321), le système lexico-sémantique bilingue comporte un certain nombre de composantes déterminant l'apparence visuelle des mots (orthographe), leur apparence sonore (phonologie), leur sens (sémantique), leurs propriétés syntaxiques (lemmes) et enfin un système de sortie qui détermine la prononciation des formes lexicales. L'orthographe et la phonologie qui sont associées à un concept lexical varient d'une langue à l'autre, par conséquent chez les sujets bilingues ou multilingues il doit y avoir des représentations distinctes au niveau orthographique et phonologique. Pour accomplir les opérations nécessaires au cours de son fonctionnement (comme par exemple la lecture en une langue ou en une autre, ou le passage d'une langue à l'autre), le système lexico-sémantique bilingue doit être contrôlé. Pour répondre à ce type de 
questions, certains auteurs (De Bot et Schreuder 1993: 191-214; Poulisse et Bongaerts 1994: 15, 36-57; Grosjean 1997: 225-254 ; Paradis 1997: 331-354) proposent l'existence d'une régulation de l'activation des systèmes de langues dans le sens de la langue d'arrivée. En revanche, d'autres (Dijkstra et van Heuven 1998: 189-225) supposent l'intervention d'une inhibition des constituants de langue qui ne correspond pas à la langue d'arrivée.

Pour fournir des indications plus précises sur les mécanismes impliqués, Green a proposé en 1998 le modèle du contrôle inhibiteur (CI), qui met à profit le modèle de système superviseur attentionnel de Norman et Shallice (1980, 1986, 1994). Dans ce modèle, Green propose l'intervention d'un circuit de contrôle fonctionnel comportant trois sites fondamentaux de contrôle: un site exécutif servant à l'établissement et au maintien des buts, un site intervenant au niveau des schémas de tâches de langage et un site intervenant au sein même du système lexico-sémantique bilingue (au niveau du lemme). Pour parler dans telle ou telle langue ou traduire d'une langue à une autre, les sujets établissent ce que Green appelle des schémas de tâches langagières. Ce sont des schémas d'action dans le domaine du langage, qui mettent en relation les entrées et sorties du système lexico-sémantique bilingue avec ses réponses. Les schémas de langage sont en compétition entre eux, et les réponses produites à chaque échéance sont déterminées par le schéma dominant au moment correspondant. La sélection correcte d'un mot dans la langue voulue interviendrait au niveau du système lexicosémantique au moyen d'une étiquette (ou marque) de langue, et au même moment, au niveau de ce site, les compétiteurs qui permettent la sélection d'une autre langue sont inhibés.

Dans le cadre de ce modèle du contrôle inhibiteur, les schémas de tâches de langage sont extérieurs au système lexico-sémantique et entrent en compétition pour en contrôler les sorties. Par exemple, pour parler en langue A, le sujet doit inhiber le schéma correspondant à la production de mots en langue B. Il s'ensuit que, pour passer d'une langue à une autre, le schéma en cours à un moment donné doit être inhibé, et le schéma ayant été précédemment inhibé doit à présent être activé. Ce processus devrait a priori représenter un certain coût, entraînant un délai temporel pour le passage d'une langue à l'autre, ce qui fut effectivement reporté dans les travaux de Meuter et Allport (Meuter, Allport 1999: 25-40) sur le plan de la production de mots et de von Studnitz et Green (von Studnitz, Green 1997: 3-24) sur le plan de la décision lexicale. À cet égard, il est intéressant de noter que, selon ces résultats, le coût du passage vers la langue dominante est plus important, ce qui suggère que le schéma de production de mots pour cette langue est plus fortement inhibé, et nécessite donc plus de temps pour être réactivé.

Au cours d'une étude neurophysiologique par tomographie par émission de positons (TEP) ayant comme objet le passage entre deux langues et le processus de traduction réalisée par Price, Green et von Studnitz (1999: 2221-2235), des sujets bilingues allemand-anglais de haut niveau linguistique ont été suivis au cours de la lecture de séries de mots en allemand et en anglais alternativement, et dans une deuxième partie de l'expérience au cours de la traduction de mots dans les deux sens. Les résultats obtenus montrent des différences au niveau de l'activation cérébrale entre la traduction et le passage entre les deux langues, ce qui suggère des mécanismes qui diffèrent, au moins partiellement. En fait, seule la traduction était accompagnée d'une augmentation de l'activation au niveau de régions cérébrales associées à la prise 
de décision, et d'une diminution de l'activation des régions associées au sens des mots. La traduction a également augmenté l'activation de régions cérébrales associées à l'articulation. Par contre, le passage entre les deux langues a été accompagné par une augmentation de l'activation de régions cérébrales associées au recodage phonologique. Il est également intéressant de signaler le fait que, au cours de ces travaux, la traduction fut accompagnée d'une diminution de l'activation de régions associées au processus sémantique par comparaison à la lecture, indépendamment de la direction de la traduction (directe ou inverse) ou de la fréquence d'occurrence des mots utilisés dans le protocole expérimental. Ces résultats sont notamment en contradiction avec des résultats de recherches psycholinguistiques, qui suggèrent deux voies différentes pour le mécanisme de traduction de simples mots: une voie lexicale directe et une voie sémantique indirecte, cette dernière intervenant davantage en situation de traduction inverse qu'en situation de traduction directe. Comme les auteurs le font remarquer, leurs résultats montrant une indépendance partielle entre les mécanismes cognitifs sous-tendant, d'une part, la traduction et, d'autre part, le passage entre les langues méritent d'être prolongés, notamment en direction de la nature de la désactivation du système sémantique au cours de la traduction. Il serait également intéressant de comprendre comment les processus lexicaux et sub-lexicaux influent sur la durée de la traduction, et plus précisément comment le mot stimulant est représenté avant que sa production ne soit bloquée.

Je voudrais également évoquer une étude de Rinne et al. (2000: 85-88) utilisant la technique TEP et portant sur l'activité cérébrale pendant l'interprétation simultanée. Leurs résultats montrent que dans ce cas l'activité cérébrale est modifiée en fonction de la direction de la traduction, étant plus intense dans le cas d'une interprétation inverse.

L'approche expérimentale de l'activité traduisante par l'étude du fonctionnement cérébral - notamment par oculométrie et par imagerie fonctionnelle - constitue sans doute une voie prometteuse pour la recherche en traductologie. Les études déjà effectuées concernent essentiellement la traduction de séries de mots choisis selon certains critères. Un pas en avant serait de pouvoir suivre par ces techniques le déroulement de l'opération de traduction dans des conditions le plus proches possible de la réalité, en travaillant donc sur la traduction de textes en entier. Une question intéressante à cet égard concerne la manière dont est représentée dans le cerveau du traducteur la macrostructure du texte: existe-t-il une structure cognitive commune pour le texte de départ et le texte d'arrivée, ou bien deux structures cognitives distinctes, une pour le texte de départ et une autre pour le texte d'arrivée?

Les sciences cognitives constituent un moyen puissant d'investigation des processus fondamentaux impliqués en traductologie. En revanche, la problématique de l'étude de l'opération traduisante dans son ensemble constitue un paradigme intéressant et stimulant pour la recherche en neurobiologie, dans le sens qu'il concerne un niveau de complexité nettement supérieur à ce qui a été envisagé jusqu'à l'heure actuelle. Tous les éléments d'une dialectique intéressante et prometteuse pour l'avenir sont donc en place...

\section{RÉFÉRENCES}

De Bot, K. (1993) : "Word production and the bilingual lexicon", in Schreuder, R., Weltens, R. (eds), The Bilingual Lexicon, Amsterdam, John Benjamins. 
Dijkstra, T., Van Heuven, W.J.B. (1998): “The BIA-model and bilingual word recognition”, in Grainger, G.

JACoBs, A. (eds), Localist Connectionist Approaches to Human Cognition, Hillsdale (NJ), Lawrence Erlbaum Associates.

Grosjean, F. (1997): "Processing mixed language: issues, findings and models", in De Groot, A.M.B., Kroll, J.F. (eds), Tutorials in Bilingualism: Psycholinguistic Perspectives, Mahwah, Lawrence Erlbaum.

Levelt, W.J.M. (1989): Speaking: from Intention to Articulation, Cambridge, MIT Press.

Meuter, R.F.I. and A. Allport (1990) : "Bilingual Language Switching in Naming: Asymmetrical Costs of Language Selection", Journal of Memory and Language 40-1, p. 25-40.

Paradis, M. (1997): "The cognitive neuropsychology of bilingualism”, in De Groot, A.M.B., Kroll, J.F. (eds), Tutorials in Bilingualism: Psycholinguistic Perspectives, Mahwah, Lawrence Erlbaum.

Patterson, K. and C. Shewell (1987): "Speak and spell: dissociations and word-class effects", in Coltheard, M., Sartori, G. and R. Јов (eds), The Cognitive Neuropsychology of Language, London, Lawrence Erlbaum.

Poulisse, N. and T. Bongaerts (1994) : "First language in second language production", Applied Linguistics 15-1, p. 36-57.

Price, C.J., Green, D.W. and R. Von Studnitz (1999): "A functional imaging study of translation and language switching", Brain 122, p. 2221-2235.

Rinne, J.O., Tommola, J., Laine, M., Krause, B.J., Schmidt, D., Kaasinen, V., Teräs, M., Sipilä, H. and M. Sunnari (2000): "The translating brain: cerebral activation patterns during simultaneous interpreting", Neuroscience Letters 294, p. 85-88. 